\title{
Privacidad en Internet desde la perspectiva de los jóvenes de nivel preparatoria en Querétaro ${ }^{8}$
}

\author{
Ana Teresa Sepúlveda Hernández ${ }^{1}$
}

\begin{abstract}
Resumen
El objetivo de este trabajo es acercarse a conocer el rol de la privacidad en las prácticas de Internet de jóvenes de preparatoria. Teóricamente el análisis se sustenta en las proposiciones de la economía política de la comunicación. La metodología es mixta con preponderancia cualitativa. Los resultados dejan ver una brecha en el acceso a Internet entre el contexto rural y el urbano; sin embargo, la mayoría de los jóvenes usan Internet y las redes sociales digitales pero el tema de la privacidad no es una prioridad.

\section{Abstract}

The objective of this project was to explore the role of privacy in high school students' use of Internet. Theoretically speaking, this study is placed in Political Economy Communication. A mixed method was applied, from a qualitative perspective. Results show a gap between Internet access in the rural and urban context of the state of Queretaro. However, most young people use Internet and digital social networks, but they don't prioritize the topic of privacy.
\end{abstract}

\section{Palabras Clave}

Privacidad, Internet, Jóvenes, Urbano, Rural

Keywords

Privacy, Internet, Youth, Urban, Rural

*Premio de Maestría del Concurso de Trabajos Recepcionales en Comunicación CONEICC 2018. Maestría en Comunicación y Cultura Digital, Facultad de Ciencias Políticas y Sociales, Universidad Autónoma de Querétaro. Directora de tesis: Dra. Miriam Herrera Aguilar.

*Autor para correspondencia: anhinsepu16@gmail.com

\section{Antecedentes, planteamiento del problema y fundamentación teórica}

En las últimas décadas se ha dado un acelerado desarrollo de las Tecnologías de la Información y de la Comunicación (TIC), incluido el sistema de Internet. De acuerdo con el informe de The State of Broadband 2016: Broadband Catalyzing Sustainable Development (2016) de Naciones Unidas, fundado en información de la Unión Internacional de Telecomunicaciones (UIT), mientras que en 2015 existían 3.200 millones de usuarios de la red en el mundo, en 2016 incrementarían a 3.500 millones (47\% de la población mundial). En México, la Encuesta Nacional sobre Disponibilidad y Uso de Tecnologías de la Información en los Hogares (ENDUTIH) 2016 deja ver que 65.5 millones de personas (59.5\% de la población) utilizan Internet y 60.6 millones usan un llamado teléfono "inteligente" (INEGI, 2016).

En este contexto, los jóvenes se han proyectado como los principales usuarios de estas innovaciones. Con base en la misma ENDUTIH 2016, el 85.5\% de la población de 12 a 17 años son usuarios de Internet, así como el $85.0 \%$ de quienes tienen entre 18 y 24 años (INEGI, 2016). Entre las principales actividades de los usuarios de Internet se tiene la de comunicación, con $88.9 \%$; la búsqueda de información, con $84.5 \%$; el consumo de contenidos audiovisuales, con $81.9 \%$; el entretenimiento, con $80.1 \%$ y el acceso a redes sociales con $75.8 \%$ (INEGI, 2016). 
Como se puede observar, actualmente la mayoría de los jóvenes, incluidos los que habitan el estado de Querétaro, se encuentran en contacto con las TIC e Internet, lo que los sumerge en un mundo infinito de circulación de información; misma que abarca diferentes dimensiones tanto de forma como de fondo. El uso de la red ha dejado ver sus grandes ventajas como medio de información y de comunicación para satisfacer necesidades de orden social, escolar y laboral, entre otros. Sin embargo, estas posibilidades implican desventajas que no necesariamente son discutidas entre los usuarios de Internet. Uno de los problemas que surge alrededor de la puesta en circulación de información en las diferentes plataformas de Internet es el manejo de la privacidad, tanto por parte de los usuarios como de las empresas que gestionan la red.

En este trabajo, la noción de privacidad toma como punto de partida "el sentido común que contrapone lo privado a lo público" (Ariès y Duby, 1987: 10), tomando en cuenta que este se modifica a través del tiempo. Si bien el tratamiento de tal noción tiene ya una larga historia, con la aparición de los medios de comunicación, y más recientemente Internet y sus diferentes plataformas, incluidas las redes sociales digitales, adquiere nuevos significados. Al respecto, Lévy menciona que "cuando una persona, una colectividad, un acto, una información se virtualizan, se colocan 'fuera de ahí', se desterritorializan" (1999 [1995]: 14); quedan expuestas.

De lo anterior se desprende el cuestionamiento: ¿Qué acceso tienen los jóvenes de nivel preparatoria de diferentes contextos sociodemográficos, en el estado de Querétaro, a las Tecnologías de Información y Comunicación (TIC)? ¿Cuáles son las principales actividades que estos jóvenes realizan en Internet? ¿Qué ventajas y desventajas identifican los jóvenes en los usos de Internet? ¿Qué rol juega la privacidad en las prácticas de estos jóvenes con Internet?

Teóricamente este planteamiento se sustenta en las proposiciones de la economía política de la comunicación; misma que, según Mattelart (1997), se gesta en los años setenta con base en el desequilibrio que se da a nivel internacional en los flujos de información y de los productos culturales. En esta línea, Rodrigo Alsina precisa que esta propuesta "centra su búsqueda en la red de intereses económicos que determinan el funcionamiento de los medios de comunicación, pero también se plantea cómo construyen una ideología a partir de sus discursos y cómo esto incide en la sociedad" (2001: 201-202).

Por su parte, Mosco (2011) señala que "la economía política ha tendido a prestar una atención considerable a la descripción y el análisis del capitalismo, un sistema que, en resumidas cuentas, convierte a los recursos, como los trabajadores, las materias primas, la tierra y la información, en mercancías comerciables que producen un beneficio para aquellos que invierten capital en el sistema" (2011: 77). Este autor propone tres procesos fundamentales para el estudio de la economía política: la mercantilización, la espacialización y la estructuración (Mosco, 2006).

En la misma línea, Mattelart y Vitalis (2015) especifican que existen grandes monopolios en Internet como Google, Facebook, Amazon, Apple y Microsoft, que dominan la red, el intercambio y los flujos de información, por lo cual ejercen cierta influencia en los internautas que dependen cada vez más de los servicios ofrecidos por estas corporaciones. "Una situación de monopolio conlleva también graves inconvenientes para la vida privada: "Dada la calidad de los servicios ofrecidos por las empresas dominantes, que se benefician de los mayores efectos de red, los internautas llegan a convertirse en dependientes de aquéllas" (Mattelart y Vitalis, 2015: 179).

Por último, Mosco apunta que la economía política de la comunicación ha estudiado la clase social a partir de las desigualdades de los individuos, poniendo énfasis en los que tienen y los que no tienen acceso a los medios de comunicación. Lo anterior, no sin precisar que no es el único elemento para entender la vida social (Mosco, 2006).

Lo anterior justifica la discusión sobre la privacidad y el derecho a salvaguardarla; cuestión que se hace cada vez más compleja no sólo por el creciente desarrollo y uso de las TIC, sino por los intereses tanto políticos como económicos que rodean los datos que ahí circulan. Este marco teórico permite formular la siguiente hipótesis de trabajo: los jóvenes de nivel preparatoria, de diferentes contextos sociodemográficos del estado de Querétaro, realizan diferentes prácticas en Internet en las que su privacidad es vulnerable y no necesariamente son conscientes de ello. 


\section{Una metodología para indagar sobre la privacidad en Internet}

La perspectiva metodológica desde donde se aborda la problemática es mixta predominantemente cualitativa. También se propone un enfoque comparativo entre los distintos casos de estudio. La selección de estos últimos se lleva a cabo con base en el criterio de suficiencia comparativa de Orozco y González, que propone que el número de casos a estudiar se define "a través de comparaciones en la búsqueda de lo distintivo, no es el de muestreo estadístico, sino el de distinción de procesos en la singularidad" (2012: 137). Los casos de estudio son los siguientes:

1. Escuela urbana con población mayormente favorecida (E-U-FAV): Preparatoria Tecnológico de Monterrey, Campus Querétaro.

2. Escuela urbana con población medianamente favorecida (E-U-MFAV): Escuela de Bachilleres "Salvador Allende", Universidad Autónoma de Querétaro, Preparatoria Sur.

3. Escuela urbana con población mayormente desfavorecida (E-U-DESFAV): Escuela de Bachilleres "Salvador Allende", Universidad Autónoma de Querétaro, Preparatoria Norte.

4. Escuela rural con población mestiza (E-R-MES): Escuela de Bachilleres "Salvador Allende", Universidad Autónoma de Querétaro, Campus Colón.

5. Escuela rural con población mestiza e indígena (E-R-IND): Plantel 28 del Colegio de Bachilleres (COBAQ), San Ildefonso, Amealco, Qro.

La selección de los grupos participantes en cada plantel, para llevar a cabo la recolección de datos, se hizo al azar. El trabajo de campo pudo realizarse gracias a la buena disponibilidad de los directores de las preparatorias y de los docentes que cedieron el tiempo de su clase. La unidad de análisis son los jóvenes, hombres y mujeres, que cursan estudios de nivel medio superior en diferentes contextos sociodemográficos del estado de Querétaro. Con la selección realizada se pretende contar con los "distintos" perfiles de los preparatorianos en la entidad.
En cuanto a la recolección de datos, en un primer momento se aplicó un cuestionario de preguntas cerradas y abiertas a todos los alumnos de cada grupo; las preguntas se precisan al momento de exponer los resultados. En un segundo momento, con los mismos jóvenes, se lleva a cabo un taller para reflexionar sobre las ventajas, desventajas y problemáticas que ofrece Internet, abordando específicamente el tema de las redes sociales digitales y poniendo especial atención en el tema de la privacidad; el cuestionamiento se precisa también en la presentación de los resultados.

\section{Resultados y discusión}

\subsection{El perfil de los jóvenes participantes y sus posibilidades de acceso a las TIC y a Internet}

Los jóvenes participantes, inscritos en las diferentes escuelas estudiadas, tienen entre 14 y 21 años de edad, la mayoría se ubica entre los 15 y 17; el promedio general es de 16.13 años.

Respecto al acceso que estos jóvenes de diferentes contextos sociodemográficos en el estado de Querétaro tienen a las TIC y a Internet, los resultados dejan ver diferencias significativas entre los que habitan en la zona urbana y quienes viven en la rural. Como se observa en la Figura 1 , los alumnos de las escuelas preparatorias ubicadas en la ciudad, independientemente del tipo de población, tienen mejor acceso que los de las escuelas preparatorias del campo.

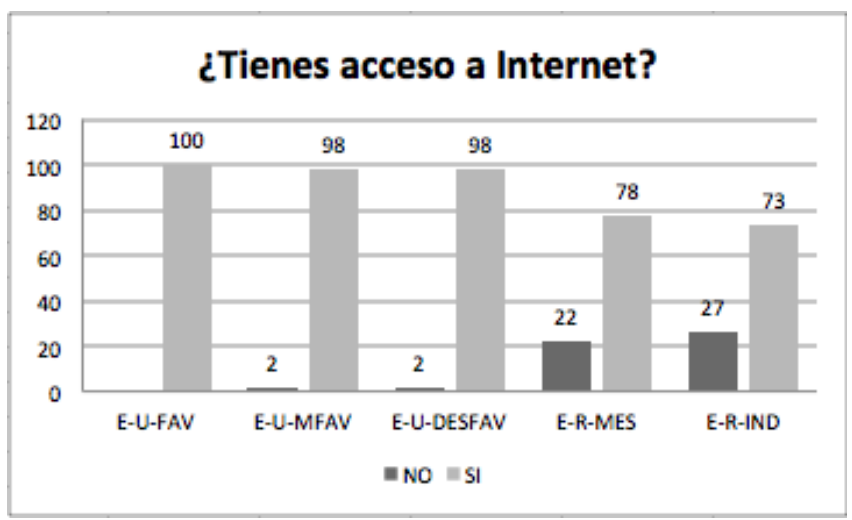

Figura 1. Acceso a Internet. Fuente: Elaboración propia con base en los resultados obtenidos en el estudio.

Especialmente en la escuela rural con población mestiza e indígena, los jóvenes manifiestan no contar con acceso a Internet. Aunado a los datos cuantitativos, resultado del 
cuestionario, en la parte cualitativa del trabajo de campo, durante el taller, algunos participantes externan que la señal de Internet no llega a sus comunidades y que el servicio es interrumpido constantemente en la escuela. En la misma línea, el personal de la institución señala que sólo "por ratos" tienen acceso a Internet a pesar de contar con la infraestructura.

En este caso, se puede afirmar que existe una brecha digital entre la ciudad y el campo en el Estado de Querétaro. Estos resultados sostienen las proposiciones de Castells cuando afirma que "la sociedad red funciona sobre la base de una lógica binaria de inclusión-exclusión, cuyas fronteras varían en el tiempo" (2006: 50). También respaldan las de Crovi (2010) cuando señala que tal fisura se relaciona con el lugar de residencia, en este caso, la zona rural; y las de Tello (2008) cuando afirma que la brecha digital en cada país incrementa las exclusiones de los grupos más desfavorecidos, entre ellos los pueblos indígenas.

Referente al lugar donde los participantes se conectan a Internet, como se muestra en la Figura 2, nuevamente aparecen similitudes entre quienes asisten a las escuelas urbanas; la casi totalidad de los actores lo hacen principalmente desde el hogar $\mathrm{y}$, en segundo lugar, desde la red wi-fi de la escuela a la que asisten. Donde hay una distinción significativa entre estos tres planteles es en la posibilidad que tienen los alumnos de disponer de una red móvil propia; ya que $90 \%$ de quienes asisten a la escuela con población mayormente favorecida, privada, cuenta con este servicio, mientras que, de los jóvenes que asisten a las públicas, sólo dos tercios acceden a tal servicio.

En lo que respecta a las escuelas rurales, ambas públicas, sus alumnos tienen aún menor acceso al servicio de Internet en el hogar. Principalmente en la escuela rural con población mestiza e indígena, dos de cada 10 acceden al servicio desde casa y más de ocho de cada 10 jóvenes deben acudir a un cibercafé para tener acceso a la red. Otro aspecto distinguible es el deficiente servicio de wi-fi de esta escuela, de ahí que la mayoría se conecte en la sala de cómputo. En la escuela rural con población mestiza, uno de cada 10 alumnos señaló hacer uso de Internet desde la casa de amigos o familiares, la biblioteca, el trabajo, jardines públicos, la calle o la casa de un vecino.

Por último, alrededor del $50 \%$ del total de los participantes en el estudio, independientemente de su contexto, tienen acceso a Internet desde la conexión inalámbrica de un lugar público.

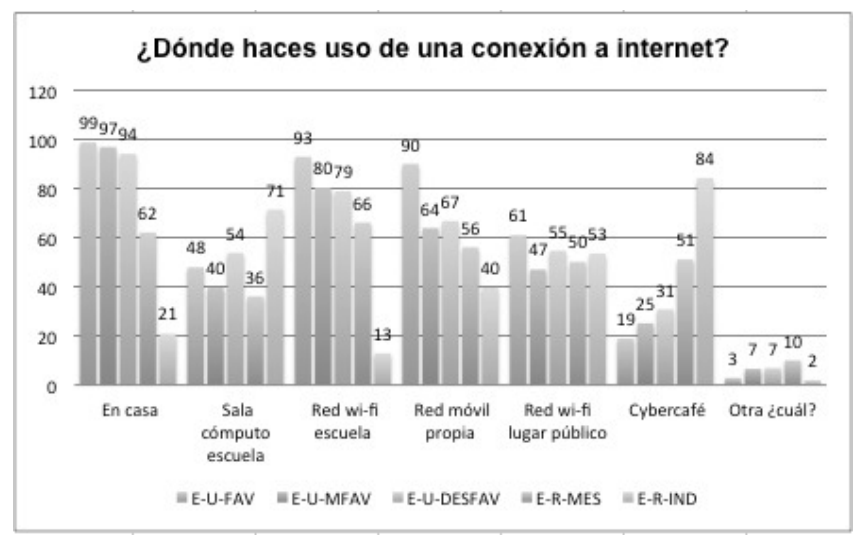

Figura 2. Lugares de conexión a Internet. Fuente:

Elaboración propia con base en los resultados obtenidos en el estudio.

Así, estos resultados reflejan la existencia de una brecha digital en la estructura social. En cuanto a los dispositivos que los jóvenes participantes utilizan para tener acceso a Internet, se encuentran diferencias significativas. El teléfono celular es el aparato que usa la mayoría de los alumnos de las diferentes escuelas preparatorias; no obstante, debe notarse que sólo tres cuartas partes de la escuela rural con población mestiza e indígena lo utiliza, mientras que en el resto de los planteles la totalidad o una gran mayoría puede hacer uso de este. Al mismo tiempo, el uso de la computadora sigue siendo importante para los alumnos de las escuelas objeto de estudio.

Con respecto al tiempo que los participantes en el estudio permanecen conectados a Internet, hay una relación directa entre las características sociodemográficas de la población y el tiempo de conexión: los alumnos que pertenecen a poblaciones más favorecidas y habitan la ciudad tienen la posibilidad de permanecer más horas conectados a Internet, de 40 a 49 horas a la semana y, viceversa, quienes habitan el campo se conectan entre 14 y 33 horas, este último dato correspondiente a la escuela con población mestiza e indígena.

Como se puede constatar con base en los resultados expuestos, el acceso que tienen los jóvenes de nivel preparatoria del estado de Querétaro, participantes en el estudio, a las Tecnologías de Información y Comunicación (TIC) y a Internet, varía de acuerdo con el contexto sociodemográfico en el que se desarrollan: los alumnos 
pertenecientes a las escuelas con población mayormente favorecida o ubicadas en el contexto urbano tienen más posibilidades de acceder a una conexión a Internet -tanto en lo que concierne a la versatilidad de lugares, a los diferentes dispositivos para hacerlo y al tiempo de conexiónque los pertenecientes a las escuelas rurales. La distinción urbano-rural sigue siendo determinante en el acceso que los jóvenes tienen a Internet y las poblaciones indígenas sufren una doble marginación.

\section{2 ¿Qué hacen los jóvenes de diferentes contextos sociodemográficos en la red?}

Entre las actividades que los participantes realizan en Internet, en primer lugar, están las cuestiones escolares -hacer tareas e investigaciones-; en segundo lugar, se tiene el uso de redes sociales digitales - principalmente Facebook y Whatsapp- y en tercer lugar las actividades de entretenimiento -escuchar música; ver videos, películas o series y jugar videojuegos.

De las actividades que destacan entre los participantes de las escuelas rurales están las "descargas" de información; mismas que se refieren a artículos, libros o cuestiones de entretenimiento -música, videos, series, juegos, etc.-Como se puede notar, dado que no cuentan con una conexión a Internet permanente, los alumnos de ambas escuelas se ven obligados a hacer uso de espacios públicos para acceder a este medio; por esta razón, su lógica de conectividad varía, la consulta en línea se sustituye por una descarga online y una consulta offline.

En lo que respecta a las principales redes sociales digitales que los jóvenes participantes dicen utilizar, en las escuelas urbanas y rurales, como se muestra en la Figura 3, están, en primer lugar, la red social Facebook, en segundo Whatsapp y en tercero Instagram. Cabe mencionar que las tres redes sociales digitales son gratuitas y propiedad de la empresa Facebook.

Cada una de las redes sociales digitales mencionadas cuenta con diversos servicios para la comunicación. En este marco, además de Facebook, Whatsapp e Instagram, otras redes utilizadas por los jóvenes, principalmente los de las escuelas urbanas, son: Ask, plataforma para hacer preguntas de manera anónima; Blackboard, enfocada a la educación y al aprendizaje; Pinterest, para compartir imágenes; Skype,

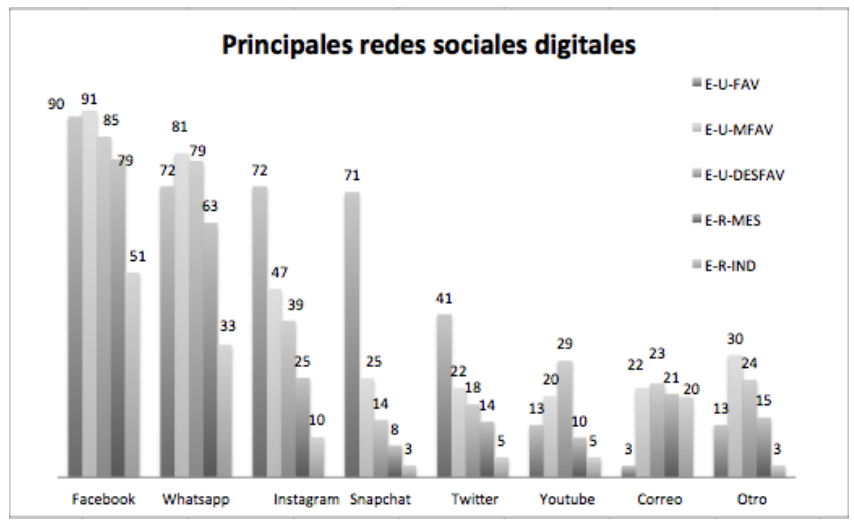

Figura 3. Principales redes sociales digitales usadas por los participantes. Fuente: Elaboración propia con base en los resultados obtenidos en el estudio.

para hacer video llamadas; Spotify, plataforma para escuchar música; Twitch, servicio de streaming enfocado a los videojuegos; Wattpad, aplicación para compartir historias; We Heart it, plataforma para compartir imágenes.

Todas estas redes sociales digitales ofrecen servicios de manera gratuita, aspecto que, como mencionan Mattelart y Vitalis (2015), hacen que el usuario olvide la dimensión comercial que la empresa tiene al recolectar sus datos personales. Aunado a lo anterior, en general, los jóvenes participantes afirman no leer las políticas de utilización de los datos, ya que lo importante para ellos es poder hacer uso de las diversas plataformas.

\subsection{Ventajas y desventajas identificadas por los jóvenes en el uso de Internet}

De manera general, las principales ventajas que los jóvenes encuentran al usar Internet es que es un medio de comunicación y de información que sirve para todo tipo de actividades incluyendo las de interacción social. Una ventaja identificada específicamente por los participantes de la zona urbana es la rapidez y facilidad de uso; mientras que los de la zona rural casi no hacen mención de tales beneficios. Un par de jóvenes de la escuela rural con población mestiza e indígena manifiestan "no saber" cuáles son los beneficios de Internet y uno más afirma: "son respuestas que aún no conozco". Como se puede observar, las ventajas de Internet no benefician a todos los jóvenes participantes. 
En cuanto a las desventajas, la mayoría de los participantes de las diferentes escuelas casos de estudio identifican como relevantes la pérdida de tiempo, la adicción a estar conectado a Internet y sus redes sociales digitales y la distracción que les impide terminar tareas. De manera específica, casi una cuarta parte de los jóvenes de la escuela urbana con población mayormente favorecida identifican como desventaja el aislamiento; parece que el hecho de ser el grupo que dispone de más dispositivos y espacios para la conexión a la red lo lleva a pasar más tiempo "capturado" por la red. Por su parte, los jóvenes de las escuelas rurales encuentran desventajas relacionadas con la infraestructura, así: "Internet es muy lento", "no llega a las comunidades" o "es costoso."

Los resultados muestran uniformidad en la percepción que los jóvenes sujetos de estudio tienen sobre las ventajas de Internet; sin embargo, es relevante identificar las numerables desventajas que encuentran, a veces en diferentes proporciones, en los distintos contextos. Estos datos rompen con la postura determinista que sólo otorga bondades al medio y muestra que los jóvenes son conscientes de que el "uso intensivo que hacen de Internet plantea muchas incógnitas sobre cómo influirá en sus relaciones sociales, familiares, en su capacidad de percepción y de expresión" (Arribas e Islas, 2009: 7).

En lo que respecta a la pérdida de privacidad como desventaja de la red, sólo es mencionada por una sexta parte de los alumnos de la escuela urbana con población mayormente favorecida y una muy mínima parte del resto de los jóvenes.

También para identificar si el tema de la privacidad está presente en las percepciones de los participantes frente al uso de Internet, se les preguntó si consideraban necesaria o no una mayor regulación de Internet y por qué. La mayoría de los jóvenes consideran que debe haber mayor regulación, principalmente en las escuelas rurales, donde menos cuentan con conexión a la red.

Al preguntar el porqué de su respuesta, ya sea positiva o negativa, solamente cuatro participantes justifican la necesidad de mayor regulación aludiendo al tema de la privacidad: un alumno de la escuela con población mayormente favorecida afirma que "hay muchos problemas relacionados con la privacidad en el medio social"; dos alumnas de la escuela con población medianamente favorecida, señalan respectivamente que "personas tontas no cuidan su privacidad" y "mi vida empieza a perder privacidad"; por último, un joven de la escuela con población mayormente desfavorecida señala que "la privacidad es invadida". Por el contrario, otro alumno de la escuela con población mayormente favorecida considera que no debe haber mayor regulación debido, precisamente, a que "violaría mi privacidad y limitaría el contenido que se encuentra en la red". En cuanto a los participantes de las escuelas rurales, ninguno hace mención explícita sobre el tema de la privacidad en Internet.

Estos resultados dejan ver que, si bien el tema de la privacidad en Internet está presente entre los participantes, no es lo que más les inquieta. Para profundizar al respecto, desde un enfoque estrictamente cualitativo, en el siguiente apartado se aborda la perspectiva de los jóvenes participantes sobre la privacidad en Internet.

\subsection{La privacidad como preocupación del uso de la red}

\subsection{1 ¿Leemos los términos de uso del servicio de redes sociales?}

Como ya se ha mostrado, la gran mayoría de los jóvenes participantes en el estudio tienen acceso a Internet, la casi totalidad de quienes asisten a las escuelas urbanas y alrededor de tres cuartas partes de quienes asisten a las rurales. Otro resultado importante para el objeto de estudio es que, después de los usos de tipo escolar, un uso ampliamente respaldado es el de las redes sociales; ya que, a excepción de la escuela rural con población mestiza e indígena donde seis de cada diez las usa, en el resto de los planteles ocho de cada 10 alumnos están inscritos en una o más.

En este contexto, resulta pertinente proponer un diálogo a partir del siguiente cuestionamiento: ¿Qué implica el uso de redes sociales? ¿Leemos los términos del contrato? ¿Qué dicen? ¿Qué ventajas y desventajas tienen? ¿Hemos tenido algún problema con su uso? ¿Nos hemos sentido incómodos por algún tipo de información que se comparte en redes sociales? ¿Por qué? Como se observa, en tal cuestionamiento no se hace explícita la problemática de la privacidad, no obstante, en las experiencias expuestas por los jóvenes, esta se revela.

Alrededor de las primeras cuatro cuestiones, la mayoría de los jóvenes en los diferentes planteles admite no leer los términos de uso del servicio y aseguran que "nadie los lee", 
que no les interesa hacerlo por ser "demasiado extensos" y “aburridos". Sin embargo, de manera general, saben de qué se trata.

En la escuela preparatoria con población mayormente favorecida, algunos plantean que "seguramente viene algo que tiene que ver con la privacidad", otro agrega que la red social Facebook "tiene derecho a utilizar tus fotos que hayas publicado para su propio beneficio" (E-U-FAV-M1) ${ }^{1}$, otros tantos coinciden con este tipo de intervenciones asumiendo que los usuarios necesariamente dan su autorización y con ello la empresa tiene acceso a toda la información que se comparte.

En la escuela con población medianamente favorecida, casi todos expresan a coro que no leen los términos del servicio; específicamente, tres de ellos aseguraron que no les importaba y uno dijo que "los había leído de manera incompleta porque se me metió un virus" (E-U-MFAV-M1). Enseguida se leen opiniones representativas de este plantel:

Sólo le das aceptar. (E-U-MFAV-M1) Una vez mi hermano quería bajar un juego y le dije que lo bajara: "aunque tengas que venderle el alma al diablo, así que sólo dale aceptar”. (E-U-MFAVF1) Sólo te resignas. (E-U-MFAV-F1)

En cuanto a los alumnos de la escuela con población mayormente desfavorecida, aparecen más excepciones al rechazo de la lectura de tales términos y sus aportes son más variados: cuatro participantes hacen alusión a las prohibiciones sobre cuestiones ilegales en el uso de redes sociales, como la publicación y comercialización de pornografía y drogas; una dice que, "a veces, cuando tengo tiempo, los leo" (E-U-DESFAV-F2); otro joven dice que "se habla sobre la sana convivencia que se debe tener dentro de estas plataformas digitales, así como ejercer la responsabilidad que implica pertenecer a una red social" (E-U-DESFAV-M2). Sólo tres participantes mencionan algo con respecto de la privacidad:

El control que tiene la empresa sobre tus datos personales. (E-U-DESFAV-M1) Los términos de

\footnotetext{
${ }^{1}$ Las diferentes aportaciones de los participantes se clasifican con la abreviatura de la escuela a la que pertenecen, a esta se agrega la $\mathrm{F}$ para femenino, la M para masculino, y el número 1, 2 y 3 para el grado de estudios que cursan.
}

privacidad, lo que puedes hacer y lo que no puedes hacer. (E-U-DESFAV-M3). Los derechos que tienen las apps para entrar a nuestra privacidad. (E-U-DESFAV-M3)

Respecto de los participantes de la escuela con población mestiza que sí tienen referentes sobre los términos de servicio de las redes sociales, cinco hacen alusión a la información solicitada - como dar acceso a tu ubicación geográfica, nombre, edad, sexo, fecha de nacimiento- y a la necesidad de aceptar que esta sea usada por terceros. Sólo tres jóvenes refieren términos y condiciones específicos relacionados con la privacidad, enseguida se citan dos de ellos:

Cuando aceptas, te dice que le das acceso a tu computadora y que van a recopilar los datos. (E-R-MES-M1) Que van a tener acceso a tus imágenes y a tus contactos. (E-R-MES-F-1)

En cuanto a la escuela con población mestiza e indígena, sólo un joven se refiere a los términos y condiciones al señalar que se debe pagar un costo por usar las redes sociales, como el tiempo y el espacio que se tiene que invertir en tales plataformas.

Los resultados permiten ver que la mayoría de los jóvenes no conoce los términos de servicio de las redes sociales digitales; lo que coincide con estudios hechos anteriormente como el de Rodríguez García y Magdalena Benedito (2016). Sin embargo, quienes conocen tales condiciones, aunque sea de manera general, están dispuestos a "pagar el precio" con tal de formar parte de estas redes; independientemente de que sus datos puedan convertirse en mercancía (Mattelart y Vitalis, 2015; Mosco, 2006, 2011).

\subsubsection{Problemas identificados por los jóvenes sobre privacidad en Internet}

En lo que respecta a la segunda parte del cuestionamiento, que busca indagar si alguien ha tenido problemas con el uso de redes sociales o se ha sentido incómodo por algún tipo de información compartida en estas; el objetivo es saber qué tanto la privacidad está en las preocupaciones de los jóvenes usuarios de las redes sociales digitales. Los jóvenes voluntariamente se expresan.

Los alumnos de la escuela con población mayormente favorecida expresan el problema de la difusión de fotografías 
comprometedoras, de la violencia, de la extorsión, de la creación de perfiles falsos y de los chismes:

Mi mamá sube fotos de cuando era chiquito y me da pena. (E-U-FAV-M1) A una amiga, intentaron extorsionarla por cosas que había subido al Facebook; de hecho, tenían toda la información de donde vivía y qué hacía su familia. (E-U-FAV-F3) Una compañera hizo un Facebook falso de los chismes locales de la escuela y ahí aparecíamos mis amigas y yo; y no fue nada agradable enterarse de eso que ponían ahí. (E-U-FAV-F3).

Mientras las problemáticas de los jóvenes pertenecientes a la escuela con población medianamente favorecida se refieren a la pornografía, a la diversidad de opiniones, a las fotos comprometedoras, al envío de packs, al humor negro, al hackeo de cuentas y la información falsa que circula en la red. Por ejemplo:

A unas chavas les enviaban pornografía. (E-U-MFAV-F1) Páginas de Facebook [...] tráfico de armas o de pornografía que no son visibles. (E-U-MFAV-M1)

Por su parte, los jóvenes de la escuela con población mayormente desfavorecida hacen notar los problemas derivados de la violencia, la adicción que provoca el uso de Internet, la pérdida de referentes de la realidad, el ciberbullying, el robo de identidad, el soborno o la extorsión, la pornografía y la información indeseable. Por ejemplo, un alumno señala:

A través de Facebook, empiezan a ver la realidad desde ahí, lo ven como su mundo. (E-U-DSFAV-M1)

En cuanto a los jóvenes de la escuela con población mestiza, externan sus preocupaciones y malestares en cuanto a la violencia o insultos y la pornografía, por ejemplo:

Una vez abrí una aplicación y me salieron anuncios eróticos. (E-R-MES-M1)
Por último, los jóvenes de la escuela rural con población mestiza e indígena mencionan el ciberbullying, el hackeo, las fotos comprometedoras, la violencia, el acoso, señalan sentirse incómodos por las burlas o críticas en cuanto a su persona o aspecto físico.

Te hackean la cuenta. (E-R-IND-F1) Que te acosen sexualmente. (E-R-IND-F1)

Las preocupaciones más respaldadas en los cinco planteles son: la violencia, la pornografía y las fotografías comprometedoras; esta última ampliamente relacionada con la privacidad. Lo anterior deja ver que los participantes, si bien identifican las ventajas que ofrece la red en diferentes aspectos de su vida, también son conscientes de los riesgos que esto implica, al tiempo que se declaran víctimas de algunos abusos. Posturas que van de la mano con lo planteado por Mattelart y Vitalis (2015) en cuanto a los riesgos latentes en el uso de las TIC e Internet y de Reig (2013) cuando señala que la obsesión por los peligros que pueden presentarse en tales usos impedirían disfrutar de las experiencias que prometen estas tecnologías.

\section{Conclusiones}

En un contexto donde no todos los jóvenes de nivel preparatoria en el estado de Querétaro tienen acceso a Internet y la brecha digital tiene lugar principalmente entre el contexto urbano y rural, el tema de la privacidad en la red no se encuentra entre sus principales inquietudes, aunque cuando se aborda el tema de manera directa no son indiferentes aunado a los diferentes problemas que han presentado o detectado dentro de la red. De manera espontánea, la preocupación por su vulnerabilidad se deja ver cuando se menciona la violencia, la pornografía y la publicación de fotografías comprometedoras como desventajas de Internet, entre otras tantas.

\section{Referencias}

Ariès, P. y Duby, G. (Eds.). (1987). Historia de la vida privada. Tomo 1. Imperio romano y antigüedad tardía. Madrid: Taurus.

Arribas, A., e Islas, O. (2009, marzo-abril). Niños y jóvenes mexicanos ante internet. En Razón y Palabra, 14 (67). 
Instituto Tecnológico y de Estudios Superiores de Monterrey. Estado de México, México. Recuperado de http://www.redalyc.org/articulo.oa?id=199520725008

Castells, M. (2006). La sociedad red: una visión global. España: Alianza Editorial.

Crovi, D. (2010). Jóvenes, migraciones digitales y brecha tecnológica. En Revista Mexicana de Ciencias Políticas y Sociales, Cuestiones Contemporáneas. Recuperado de http://www.revistas.unam.mx/index.php/rmcpys/articl e/viewFile/25967/24446

Instituto Nacional de Estadística y Geografía (INEGI). Aumentan uso de Internet teléfonos inteligentes y tv digital: Encuesta Nacional sobre Disponibilidad y Uso de Tecnologías de la Información en los Hogares (ENDUTIH) 2016. Recuperado de http://www.inegi. org.mx/saladeprensa/boletines/2017/especiales/especi ales2017_03_02.pdf

Lévy, P. ([1995] 1999). ¿Qué es lo virtual? Barcelona: Paidós. Recuperado de: http://aprendeenlinea.udea.edu.co/lms /moodle/file.php/90/documentos_actividades/levy-pier re-que-es-lo-virtual.PDF

Mattelart, A. (2007 [1997]). Historia de la sociedad de la información. Barcelona: Paidós.

Mattelart, A., y Vitalis, A. (2015). De Orwell al Cibercontrol. Barcelona, España: Gedisa.

Mosco, V. (2006). La Economía Política de la Comunicación: una actualización diez años después. En Cuadernos de Información y Comunicación CIC, vol. 11, 57-79.

Mosco, V. (2011). La economía política de la comunicación: una tradición viva. En Albornoz, L. Poder Medios, Cultura. Una mirada crítica desde la economía política de la comunicación. Buenos Aires: Paidós.

Orozco, G., y González, R. (2012). Una coartada metodológica. Abordajes cualitativos en la investigación en comunicación, medios y audiencias. Serie Brújula. México DF: Tintable.

Reig, D. (2013). Describiendo al hiperindividuo, el nuevo individuo conectado. En Reig, D. y Vilchez, L. Los jóvenes en la era de la hiperconectividad: tendencias, claves y miradas. Madrid, España: Fundación Telefónica y Fundación Encuentro.

Rodrigo Alsina, M. (2001). Teorías de la comunicación. Ámbitos, Métodos y perspectivas. Universidad de Valencia; Universidad de Pompeu Fabra; Universidad Jaume I. Universidad Autónoma de Barcelona. España: Aldea Global.

Rodríguez García, L. y Magdalena Benedito, J.R. (2016). Perspectivas de los jóvenes sobre seguridad y privacidad en las redes sociales. En Icono 14, 14, (pp. 24-49). Doi: 10.7195/ri14.v14i1.885

Tello Leal, E. (2008). Las tecnologías de la información y comunicaciones (TIC) y la brecha digital: su impacto en la sociedad en México. En Revista de Universidad y Sociedad del Conocimiento RU\&SC. Iniversitat Oberta de Catalunya. Recuperado de http://www.uoc.edu/rusc /4/2/dt/esp/tello.pdf

The State of Broadband 2016: Broadband Catalyzing Sustainable Development. Broadban Commission For Sustainable Development. Recuperado de http://www.br oadbandcommission.org/Documents/reports/bb-annua lreport2016.pdf 